\title{
The Hernquist model revisited: Completely analytical anisotropic dynamical models
}

\author{
M. Baes` and H. Dejonghe
}

\author{
Sterrenkundig Observatorium, Universiteit Gent, Krijgslaan 281-S9, 9000 Gent, Belgium \\ e-mail: maarteen.baes@rug.ac.be; herwig.dejonghe@rug.ac.be
}

Received 17 June 2002 / Accepted 12 July 2002

\begin{abstract}
Simple analytical models, such as the Hernquist model, are very useful tools to investigate the dynamical structure of galaxies. Unfortunately, most of the analytical distribution functions are either isotropic or of the Osipkov-Merritt type, and hence basically one-dimensional. We present three different families of anisotropic distribution functions that self-consistently generate the Hernquist potential-density pair. These families have constant, increasing and decreasing anisotropy profiles respectively, and can hence represent a wide variety of orbital structures. For all of the models presented, the distribution function and the velocity dispersions can be written in terms of elementary functions. These models are ideal tools for a wide range of applications, in particular to generate the initial conditions for $N$-body or Monte Carlo simulations.
\end{abstract}

Key words. galaxies: kinematics and dynamics - galaxies: structure

\section{Introduction}

From a stellar dynamical point of view, the most complete description of a stellar system is the distribution function $F(\boldsymbol{r}, \boldsymbol{v})$, which gives the probability density for the stars in phase space. In this paper, we will concentrate on the problem of constructing anisotropic equilibrium distribution functions that self-consistently generate a given spherical mass density profile $\rho(r)$. In the assumption of spherical symmetry, the mass density of a stellar system can easily be derived from the observed surface brightness profile, at least if we assume that the mass-to-light ratio is constant and that dust attenuation is negligible. And as the surface brightness of a galaxy (or bulge or cluster) is fairly cheap and straightforward to observe, compared to other dynamical observables which require expensive spectroscopy, the problem we will deal with is relevant and important.

The first step in the construction of self-consistent models is the calculation of the gravitational potential $\psi(r)$, which can immediately be determined through Poisson's equation. The second step, the actual construction of the distribution function, is less straightforward. Basic stellar dynamics theory (see e.g. Binney \& Tremaine 1987) learns that steady-state distribution functions for spherical systems can generally be written as a function of binding energy and angular momentum. We hence have to determine a distribution function $F(\mathcal{E}, L)$, such that the

Send offprint requests to: $\mathrm{M}$. Baes,

e-mail: maarten.baes@rug.ac.be

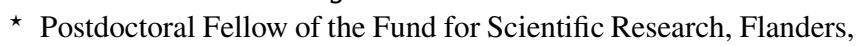
Belgium (F.W.O.-Vlaanderen). zeroth order moment of this distribution function equals the density, i.e. we have to solve the integral equation

$\rho(r)=\iiint F(\mathcal{E}, L) \mathrm{d} v$

for $F(\mathcal{E}, L)$. Hereby we have to take into account that not every function $F(\mathcal{E}, L)$ that satisfies this equation is a physically acceptable solution: an acceptable solution has to be non-negative over the entire phase space. In general, the problem of solving the integral Eq. (1) is a degenerate problem, because there are infinitely many distribution functions possible for a given potential-density pair.

Particularly interesting are models for which the distribution function and its moments can be computed analytically. Such models have many useful applications, which can roughly be divided into two classes. On the one hand, they can improve our general understanding of physical processes in galaxies in an elegant way. For example, they can serve as simple galaxy models, in which it is easy to generate the starting conditions for $\mathrm{N}$-body or Monte Carlo simulations, or to test new data reduction or dynamical modelling techniques. A quick look at the overwhelming success of simple analytical models, such as the Plummer sphere (Plummer 1911; Dejonghe 1987), the isochrone sphere (Hénon 1959, 1960), the Jaffe model (Jaffe 1983) and the Hernquist model (Hernquist 1990), provides enough evidence. On the other hand, analytical models are also useful for the detailed dynamical modelling of galaxies. For example, in modelling techniques such as the QP technique (Dejonghe 1989), a dynamical model for an observed galaxy is built up as a linear combination of components, for each of 
which the distribution function and its moments are known analytically. As a result, the distribution function and the moments of the final model are also analytical, which obviously has a number of advantages.

Unfortunately, the number of dynamical models for which the distribution function is known analytically is rather modest. Moreover, most of them consist of distribution functions that are isotropic or of the Osipkov-Merritt type, and therefore basically one-dimensional. An exception is the completely analytical family of anisotropic models described by Dejonghe (1987). These models self-consistently generate the Plummer potential-density pair, a simple yet useful model for systems with a constant density core.

During the last decade, however, it has become clear that, at small radii, elliptical galaxies usually have central density profiles that behave as $r^{-\gamma}$ with $0 \leqslant \gamma \leqslant 2.5$ (Lauer et al. 1995; Gebhardt et al. 1996). Such galaxies can obviously not be adequately modelled with a constant density core. This has stimulated the quest for simple potential-density pairs, and corresponding distribution functions, with a central density cusp. The first effort to construct such models was undertaken by Ciotti (1991) and Ciotti \& Lanzoni (1997), who discussed the the dynamical structure of stellar systems following the $R^{1 / m}$ law (Sérsic 1968), a natural generalization of the empirical $R^{1 / 4}$ law of de Vaucouleurs (1948). A major drawback of this family, however, is that the spatial density and the distribution function can not be written in terms of elementary functions (see Mazure \& Capelato 2002). A more useful family is formed by the so-called $\gamma$-models (Dehnen 1993; Tremaine et al. 1994), characterized by a density proportional to $r^{-4}$ at large radii and a divergence in the center as $r^{-\gamma}$ with $0 \leqslant \gamma \leqslant 3$. The dynamical structure of models with this potential-density pair has been extensively investigated (e.g. Carollo et al. 1995; Ciotti 1996; Meza \& Zamorano 1997), but only for isotropic or OsipkovMerritt type distribution functions. Simple analytical models with a more general anisotropy structure are still lacking.

In this paper we construct a number of families of completely analytical anisotropic dynamical models that selfconsistently generate the Hernquist (1990) potential-density pair. It is a special case of the family of $\gamma$-models, corresponding to $\gamma=1$. In dimensionless units, the Hernquist potentialdensity pair is given by

$$
\begin{aligned}
& \psi(r)=\frac{1}{1+r} \\
& \rho(r)=\frac{1}{2 \pi} \frac{1}{r(1+r)^{3}} .
\end{aligned}
$$

As the density diverges as $1 / r$ for $r \rightarrow 0$, the surface brightness $I(R)$ will diverge logarithmically for $R \rightarrow 0$. More precisely, the surface brightness profile has the form

$I(R)=\frac{1}{2 \pi} \frac{\left(2+R^{2}\right) X(R)-3}{\left(1-R^{2}\right)^{2}}$,

with $X(R)$ a continuous function defined as

$$
X(R)= \begin{cases}\left(1-R^{2}\right)^{-1 / 2} \operatorname{arcsech} R & \text { for } 0 \leqslant R \leqslant 1 \\ \left(R^{2}-1\right)^{-1 / 2} \operatorname{arcsecans} R & \text { for } 1 \leqslant R \leqslant \infty .\end{cases}
$$

The paper is organized as follows. The general theory on the inversion of the fundamental integral Eq. (1) is resumed in Sect. 2. Each of the subsequent sections is devoted to special cases of this inversion technique and the corresponding family of Hernquist models. Isotropic models are the most simple ones; Hernquist (1990) showed that, in the special case of isotropy, the distribution function and its moments can be calculated analytically. We repeat the most important characteristics of the isotropic Hernquist model in Sect. 3. In Sect. 4 we construct a one-parameter family of models with a constant anisotropy. In Sect. 5, a two-parameter family of Hernquist models is constructed by means of the Cuddeford (1991) inversion technique. These models have an arbitrary anisotropy in the center and are radially anisotropic at large radii. On the contrary, in Sect. 6, a two-parameter family is constructed that has a decreasing anisotropy profile, with arbitrary values for the anisotropy in the center and the outer halo. Finally, Sect. 7 sums up.

\section{The construction of anisotropic models}

A general discussion on the inversion of the fundamental Eq. (1), and hence on the construction anisotropic distribution functions for a given spherical potential-density pair, is presented by Dejonghe (1986). The key ingredient of the inversion procedure is the concept of the augmented mass density $\tilde{\rho}(\psi, r)$, which is a function of potential and radius, such that the condition

$\tilde{\rho}(\psi(r), r) \equiv \rho(r)$

is satisfied. The augmented mass density is in fact equivalent to the distribution function $F(\mathcal{E}, L)$ : with every augmented density $\tilde{\rho}(\psi, r)$ we can associate a distribution function $F(\mathcal{E}, L)$ and vice versa. There exist various transition formulae between these two equivalent forms of a dynamical model, amongst others a formalism that uses combined Laplace-Mellin transforms.

Besides providing a nice way to generate a distribution function for a given potential-density pair, the augmented density is also very useful to calculate the moments of the distribution function. The anisotropic moments are defined as

$\mu_{2 n, 2 m}(r)=2 \pi \iint F(\mathcal{E}, L) v_{\mathrm{r}}^{2 n} v_{\mathrm{t}}^{2 m+1} \mathrm{~d} v_{\mathrm{r}} \mathrm{d} v_{\mathrm{t}}$

where $v_{\mathrm{t}} \equiv \sqrt{v_{\theta}^{2}+v_{\phi}^{2}}$ is the transverse velocity. One can derive a relation that links the higher-order moments to the augmented mass density $\tilde{\rho}$, when written explicitly as a function of $\psi$ and $r$,

$$
\begin{aligned}
\tilde{\mu}_{2 n, 2 m}(\psi, r)= & \frac{2^{m+n}}{\sqrt{\pi}} \frac{\Gamma\left(n+\frac{1}{2}\right)}{\Gamma(m+n)} \\
& \times \int_{0}^{\psi}\left(\psi-\psi^{\prime}\right)^{m+n-1} \frac{\mathrm{d}^{m}}{\left(\mathrm{~d} r^{2}\right)^{m}}\left[r^{2 m} \tilde{\rho}\left(\psi^{\prime}, r\right)\right] \mathrm{d} \psi^{\prime} .
\end{aligned}
$$

In particular, the radial and transverse velocity dispersions can be found from the density through the relations,

$\sigma_{\mathrm{r}}^{2}(r)=\frac{1}{\rho(r)} \int_{0}^{\psi(r)} \tilde{\rho}\left(\psi^{\prime}, r\right) \mathrm{d} \psi^{\prime}$,

$\sigma_{\mathrm{t}}^{2}(r)=\frac{2}{\rho(r)} \int_{0}^{\psi(r)} \frac{\mathrm{d}}{\mathrm{d} r^{2}}\left[r^{2} \tilde{\rho}\left(\psi^{\prime}, r\right)\right] \mathrm{d} \psi^{\prime}$. 
By means of these functions, we can define the anisotropy $\beta(r)$ as

$\beta(r)=1-\frac{\sigma_{\mathrm{t}}^{2}(r)}{2 \sigma_{\mathrm{r}}^{2}(r)}$.

We will in this paper consider augmented densities which are separable functions of $\psi$ and $r$, and we introduce the notation

$\tilde{\rho}(\psi, r)=f(\psi) g(r)$.

For such models, the anisotropy can be directly calculated from the augmented density as

$\beta(r)=1-\frac{1}{g(r)} \frac{\mathrm{d}}{\mathrm{d} r^{2}}\left[r^{2} g(r)\right]$,

as a result of the formulae (8ab), (9) and (10).

\section{Isotropic models}

\subsection{Background}

The simplest dynamical models are those where the augmented density is a function of the potential only, $\tilde{\rho}=\tilde{\rho}(\psi)$. For such models, the distribution function is only a function of the binding energy, i.e. the distribution function is isotropic. In this case, the integral Eq. (1) can be inverted to find the well-known Eddington relation

$F(\mathcal{E})=\frac{1}{2 \sqrt{2} \pi^{2}} \frac{\mathrm{d}}{\mathrm{d} \mathcal{E}} \int_{0}^{\mathcal{E}} \frac{\mathrm{d} \tilde{\rho}}{\mathrm{d} \psi} \frac{\mathrm{d} \psi}{\sqrt{\mathcal{E}-\psi}}$.

For such isotropic models, we do not use the general anisotropic moments (6), but define the isotropic moments as

$\mu_{2 n}(r)=4 \pi \int F(\mathcal{E}) v^{2 n+2} \mathrm{~d} v$.

Similarly as for the anisotropic moments, we can derive a relation that allows to calculate the augmented isotropic moments from the augmented density $\tilde{\rho}(\psi)$. Indeed, they satisfy the relation (Dejonghe 1986)

$\tilde{\mu}_{2 n}(\psi)=\frac{(2 n+1) ! !}{(n-1) ! !} \int_{0}^{\psi}\left(\psi-\psi^{\prime}\right)^{n-1} \tilde{\rho}\left(\psi^{\prime}\right) \mathrm{d} \psi^{\prime}$.

In particular, we obtain an expression for the velocity dispersion profile by setting $n=1$,

$\sigma^{2}(r)=\frac{1}{\rho(r)} \int_{0}^{\psi(r)} \tilde{\rho}\left(\psi^{\prime}\right) \mathrm{d} \psi^{\prime}$

\subsection{The isotropic Hernquist model}

The isotropic model that corresponds to the potential-density pair (2ab) is described in full detail by Hernquist (1990). We restrict ourselves by resuming the most important results, for a comparison with the anisotropic models discussed later in this paper. The augmented density reads

$\tilde{\rho}(\psi)=\frac{1}{2 \pi} \frac{\psi^{4}}{1-\psi}$.
Substituting this density into Eddington's formula (12) yields the distribution function

$$
\begin{aligned}
F(\mathcal{E})= & \frac{1}{8 \sqrt{2} \pi^{3}} \\
& \times\left[\frac{\sqrt{\mathcal{E}}(1-2 \mathcal{E})\left(8 \mathcal{E}^{2}-8 \mathcal{E}-3\right)}{(1-\mathcal{E})^{2}}+\frac{3 \arcsin \sqrt{\mathcal{E}}}{(1-\mathcal{E})^{5 / 2}}\right] .
\end{aligned}
$$

Combining the density (16) with the general formula (14) gives us the moments of the distribution function,

$\tilde{\mu}_{2 n}(\psi)=\frac{3 \cdot 2^{n+9 / 2}}{(2 \pi)^{3 / 2}} \frac{\Gamma\left(n+\frac{1}{2}\right)}{\Gamma(n+5)} \psi_{2}^{n+4}{ }_{2} F_{1}(5,1 ; n+5 ; \psi)$.

For all $n \geqslant 0$, this expression can be written in terms of rational functions and logarithms. For example, for the velocity dispersions, we obtain after substitution of the Hernquist potential (2a),

$\sigma^{2}(r)=r(1+r)^{3} \ln \left(\frac{1+r}{r}\right)-\frac{r\left(25+52 r+42 r^{2}+12 r^{3}\right)}{12(1+r)}$,

in agreement with Eq. (10) of Hernquist (1990). From an observational point of view, it is very useful to obtain an explicit expression for the line-of-sight velocity dispersion. For isotropic models, the line-of-sight dispersion is easily found by projecting the second-order moment on the plane of the sky, i.e.

$\sigma_{\mathrm{p}}^{2}(R)=\frac{2}{I(R)} \int_{R}^{\infty} \frac{\rho(r) \sigma^{2}(r) r \mathrm{~d} r}{\sqrt{r^{2}-R^{2}}}$.

For the Hernquist model this yields after some algebra

$$
\begin{aligned}
I(R) \sigma_{\mathrm{p}}^{2}(R)= & \frac{1}{24 \pi\left(1-R^{2}\right)^{3}} \\
& \times\left[3 R^{2}\left(20-35 R^{2}+28 R^{4}-8 R^{6}\right) X(R)\right. \\
& \left.+\left(6-65 R^{2}+68 R^{4}-24 R^{6}\right)\right]-\frac{R}{2} .
\end{aligned}
$$

\section{Models with a constant anisotropy}

\subsection{Background}

A special family of distribution functions that can easily be generated using the technique outlined in Sect. 2 corresponds to models with a density that depends on $r$ only through a factor $r^{-2 \beta}$, i.e.

$\tilde{\rho}(\psi, r)=f(\psi) r^{-2 \beta}$.

It is well known that such densities correspond to models with a constant anisotropy (i.e. Binney \& Tremaine 1987), which can easily be checked by introducing $g(r)=r^{-2 \beta}$ in the formula (11). For a given potential-density pair, a family of models with constant anisotropy can hence be constructed by inverting the potential as $r(\psi)$, and defining

$f(\psi)=\tilde{\rho}(\psi)(r(\psi))^{2 \beta}$.

Notice that such models are not the only constant anisotropy models corresponding to a given potential-density pair, as argued in Sect. 1.5 of Dejonghe (1986). This family, however, 
is very attractive due to its relative simpleness. In particular, the corresponding distribution function is a power law in $L$, and can be found through an Eddington-like formula,

$$
\begin{aligned}
F(\mathcal{E}, L)= & \frac{2^{\beta}}{(2 \pi)^{3 / 2}} \frac{L^{-2 \beta}}{\Gamma(1-\beta) \Gamma\left(\frac{1}{2}+\beta\right)} \\
& \times \frac{\mathrm{d}}{\mathrm{d} \mathcal{E}} \int_{0}^{\mathcal{E}} \frac{\mathrm{d} f}{\mathrm{~d} \psi} \frac{\mathrm{d} \psi}{(\mathcal{E}-\psi)^{1 / 2-\beta}} .
\end{aligned}
$$

For the moments of the distribution function, the relation (7) can be simplified to

$$
\begin{aligned}
\tilde{\mu}_{2 n, 2 m}(\psi, r)= & \frac{2^{m+n}}{\sqrt{\pi}} \frac{\Gamma\left(n+\frac{1}{2}\right) \Gamma(m+1-\beta)}{\Gamma(m+n) \Gamma(1-\beta)} r^{-2 \beta} \\
& \times \int_{0}^{\psi}\left(\psi-\psi^{\prime}\right)^{m+n-1} f\left(\psi^{\prime}\right) \mathrm{d} \psi^{\prime} .
\end{aligned}
$$

In particular, the radial velocity dispersions reads

$\sigma_{\mathrm{r}}^{2}(r)=\frac{1}{f(\psi(r))} \int_{0}^{\psi(r)} f\left(\psi^{\prime}\right) \mathrm{d} \psi^{\prime}$.

\subsection{Hernquist models with a constant anisotropy}

\subsubsection{The distribution function}

Applying the formula (23) to the Hernquist potential-density pair (2ab) yields

$f(\psi)=\frac{1}{2 \pi} \psi^{4-2 \beta}(1-\psi)^{2 \beta-1}$.

Substituting this expression into the general formula (24) gives us the corresponding distribution function

$$
\begin{aligned}
F(\mathcal{E}, L)= & \frac{2^{\beta}}{(2 \pi)^{5 / 2}} \frac{\Gamma(5-2 \beta)}{\Gamma(1-\beta) \Gamma\left(\frac{7}{2}-\beta\right)} \\
& \times L^{-2 \beta} \mathcal{E}^{\frac{5}{2}-\beta}{ }_{2} F_{1}\left(5-2 \beta, 1-2 \beta ; \frac{7}{2}-\beta ; \mathcal{E}\right)
\end{aligned}
$$

This expression reduces to the isotropic distribution function (17) for $\beta=0$, as required. Whether the expression (28) corresponds to a physically acceptable distribution function for a given value of $\beta$ depends on the condition that the distribution function has to be positive over the entire phase space.

It is no surprise that the distribution function is not positive for the largest possible values of $\beta$, because models where only the radial orbits are populated can only be supported by a density profile that diverges as $r^{-2}$ or steeper in the center (Richstone \& Tremaine 1984). It turns out that the distribution function (28) is everywhere non-negative for $\beta \leqslant \frac{1}{2}$.

For all integer and half-integer values of $\beta$, the hypergeometric series in (28) can be expressed in terms of elementary functions. Very useful are half-integer values of $\beta$, because the energy-dependent part of the distribution function can then be written as a rational function of $\mathcal{E}$. For integer values of $\beta$, the hypergeometric series can be written as a function containing integer and half-integer powers of $\mathcal{E}$ and $1-\mathcal{E}$ and a factor $\arcsin \sqrt{\mathcal{E}}$, similar to the isotropic distribution function (17).
The limiting model $\beta=\frac{1}{2}$ is particularly simple. It has an augmented density that is a power law of potential and radius,

$\tilde{\rho}(\psi, r)=\frac{1}{2 \pi} \frac{\psi^{3}}{r}$,

and the corresponding distribution function simply reads

$F(\mathcal{E}, L)=\frac{3}{4 \pi^{3}} \frac{\mathcal{E}^{2}}{L}$

This model is a special case of the generalized polytropes discussed by Fricke (1951) and Hénon (1973).

In Fig. 1 we compare the distribution functions of the radial model with $\beta=\frac{1}{2}$ and the tangential model with $\beta=-2$ with the distribution function of the isotropic Hernquist model. The distribution functions are shown by means of their isoprobability contours in turning point space, which can easily be interpreted in terms of orbits. Compared to the isotropic model, the radial model prefers orbits on the upper left side of the diagram, with an apocenter much larger than the pericenter, i.e. elongated orbits. The isoprobability contours of tangential models on the other hand lean towards the diagonal axes where pericenter and apocenter are equal, i.e. nearly-circular orbits are preferred.

\subsubsection{The velocity dispersions}

By means of substituting the expression (27) into the general formula (7), we can derive an analytical expression for all moments of the distribution function,

$$
\begin{aligned}
\tilde{\mu}_{2 n, 2 m}(\psi, r)= & \frac{2^{n+m-1}}{\pi^{3 / 2}} \frac{\Gamma(5-2 \beta) \Gamma\left(n+\frac{1}{2}\right) \Gamma(m+1-\beta)}{\Gamma(m+n+5-2 \beta) \Gamma(1-\beta)} \\
& \times r^{-2 \beta} \psi^{m+n+4-\beta} \\
& \times{ }_{2} F_{1}(5-2 \beta, 1-2 \beta ; m+n+5-2 \beta ; \psi) .
\end{aligned}
$$

We are mainly interested in the velocity dispersions, which can be conveniently written by means of the incomplete Beta function (Abramowitz \& Stegun 1972),

$\sigma_{\mathrm{r}}^{2}(r)=r^{1-2 \beta}(1+r)^{3} \mathrm{~B}_{\frac{1}{1+r}}(5-2 \beta, 2 \beta)$.

For all anisotropies $\beta<\frac{1}{2}$, the radial dispersion equals zero in the center of the galaxy, rises until a maximum and then decreases again towards zero for $r \rightarrow \infty$. The asymptotic behavior for $r \gg 1$ is

$\sigma_{\mathrm{r}}^{2}(r) \approx \frac{1}{5-2 \beta} \frac{1}{r}+\cdots$

The expression (32) can be written in terms of elementary functions for all $\beta$ with $4 \beta$ an integer. For the integer and half-integer values of $\beta$, the expression involves polynomials in $r$ and a factor $\ln (1+1 / r)$, very analogous to the expression (19) of the isotropic Hernquist model. For the quarter-integer values of $\beta$, it contains polynomials and square roots in $r$ and a factor $\operatorname{arccotg} \sqrt{r}$. 

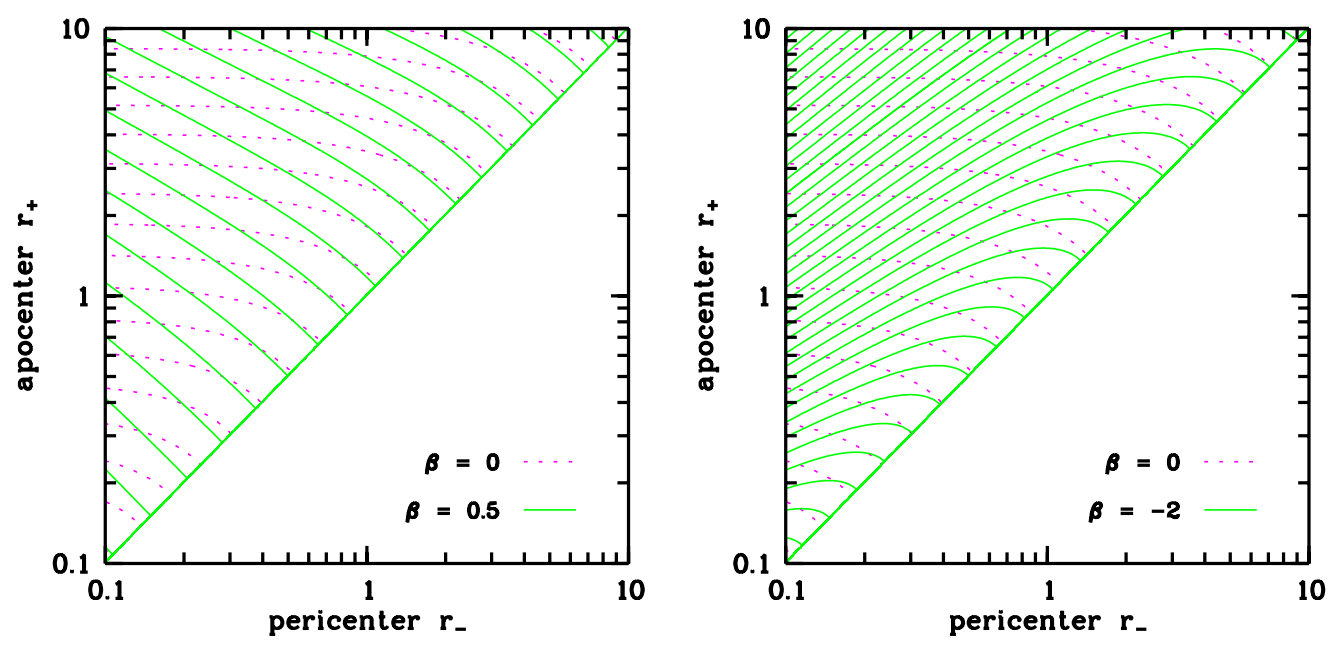

Fig. 1. The distribution function of the Hernquist models with a constant anisotropy, represented as isoprobability contours in turning point space. The distribution functions in solid lines represent a radial model with $\beta=\frac{1}{2}$ (left panel) and a tangential model with $\beta=-2$ (right panel). The dotted contour lines in both panels correspond to the isotropic Hernquist model.
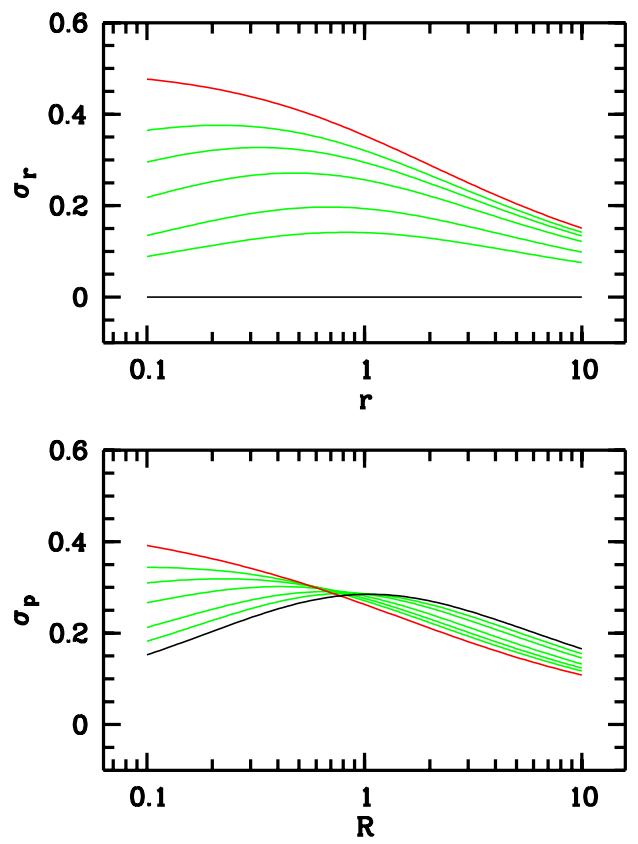

Fig. 2. The velocity dispersion of the Hernquist models with a constant anisotropy. The upper and lower panels show the radial velocity dispersions $\sigma_{\mathrm{r}}(r)$ and the line-of-sight velocity dispersion $\sigma_{\mathrm{p}}(R)$. The profiles are shown for different values of the anisotropy parameter $\beta$ : plotted are $\beta=\frac{1}{2}, \frac{1}{4}, 0,-\frac{1}{2},-2,-5$ and the limit case $\beta \rightarrow-\infty$.

Particular cases are the models that correspond to the most radial and tangential distribution functions. On the one hand, the limit case $\beta=\frac{1}{2}$ has the simple velocity dispersion profiles

$\sigma_{\mathrm{r}}^{2}(r)=\sigma_{\mathrm{t}}^{2}(r)=\frac{1}{4} \frac{1}{1+r}$.

Particular about this dispersion profile is that it assumes a finite value in the center. On the other side of the range for possible anisotropies, we can consider the limit $\beta \rightarrow-\infty$, which corresponds to a model with purely circular orbits. For such a model, the radial dispersion is of course identically zero, whereas the transverse velocity dispersion is the circular velocity corresponding to the Hernquist potential,

$\sigma_{\mathrm{t}}^{2}(r)=v_{\mathrm{c}}^{2}(r)=\frac{1}{2} \frac{r}{(1+r)^{2}}$.

In the top panel of Fig. 2 we plot the radial velocity dispersion profile for various models with a different anisotropy $\beta$. Both at small and large radii, the radial dispersion is a decreasing function of $\beta$, as expected.

The line-of-sight velocity dispersion for anisotropic models is found through the formula

$\sigma_{\mathrm{p}}^{2}(R)=\frac{2}{I(R)} \int_{R}^{\infty} \frac{\rho(r) \sigma_{\text {los }}^{2}(r, R) r \mathrm{~d} r}{\sqrt{r^{2}-R^{2}}}$,

where $\sigma_{\text {los }}(r, R)$ is the velocity dispersion at the position $r$ on the line of sight $R$ in the direction of observer. It is a linear combination of the radial and transverse velocity dispersions in this point,

$\sigma_{\mathrm{los}}^{2}(r, R)=\left(1-\frac{R^{2}}{r^{2}}\right) \sigma_{\mathrm{r}}^{2}(r)+\frac{R^{2}}{2 r^{2}} \sigma_{\mathrm{t}}^{2}(r)$.

We can equivalently write

$\sigma_{\mathrm{p}}^{2}(R)=\frac{2}{I(R)} \int_{R}^{\infty}\left[1-\beta(r) \frac{R^{2}}{r^{2}}\right] \frac{\rho(r) \sigma_{\mathrm{r}}^{2}(r) r \mathrm{~d} r}{\sqrt{r^{2}-R^{2}}}$.

For the general Hernquist models with a constant anisotropy, the integration (38) cannot be performed analytically. But for all integer and half-integer $\beta$ 's, $\sigma_{\mathrm{p}}^{2}(R)$ can be expressed in terms of polynomials and the function $X$, defined in Eq. (4). For example, for the limit model $\beta=\frac{1}{2}$ we obtain after some algebra

$$
\begin{aligned}
I(R) \sigma_{\mathrm{p}}^{2}(R)= & \frac{1}{48 \pi\left(1-R^{2}\right)^{3}} \\
& \times\left[3\left(4-14 R^{2}+35 R^{4}-28 R^{6}+8 R^{8}\right) X(R)\right. \\
& \left.-\left(28-57 R^{2}+68 R^{4}-24 R^{6}\right)\right]+\frac{R}{4}
\end{aligned}
$$


For the other limit model, the one with only circular orbits, we find

$$
\begin{aligned}
& I(R) \sigma_{\mathrm{p}}^{2}(R)=\frac{R^{2}}{48 \pi\left(1-R^{2}\right)^{4}} \\
& \times\left[-\left(120-120 R^{2}+189 R^{4}-108 R^{6}+24 R^{8}\right) X(R)\right. \\
& \left.\quad+\left(154-117 R^{2}+92 R^{4}-24 R^{6}\right)\right]+\frac{R}{4},
\end{aligned}
$$

in agreement with Hernquist (1990).

In the bottom panel of Fig. 2 we plot the line-of-sight dispersion profiles for a number of different values of $\beta$. The behavior of the individual profiles is analogous to the spatial dispersion profiles: except for the $\beta=\frac{1}{2}$ model, which has a finite central dispersion, the $\sigma_{\mathrm{p}}$ profiles start at zero in the center, rise strongly until a certain maximum and then decrease smoothly towards zero at large projected radii. The behavior for $R \gg 1$ can be quantified if we introduce the asymptotic expansion (33) into the formula (38),

$\sigma_{\mathrm{p}}^{2}(R) \approx \frac{8}{15 \pi}\left(\frac{5-4 \beta}{5-2 \beta}\right) \frac{1}{R}+\cdots$

The dependence of the line-of-sight dispersion as a function of the anisotropy is depends strongly on the projected radius: at small projected radii, $\sigma_{\mathrm{p}}$ decreases with increasing $\beta$, whereas for large radii, $\sigma_{\mathrm{p}}$ increases with increasing $\beta$. In other words, radial models have a larger central and a smaller outer lineof-sight dispersion than their tangential counterparts. This is a direct consequence of the weight of the radial and transversal velocity dispersions in the linear combination (37): at small projected radii, the radial dispersion contributes the dominant term, whereas for the outer lines of sight, the transversal dispersion term dominates.

\section{Models with increasing anisotropy}

\subsection{Background}

Osipkov (1979) and Merritt (1985) developed an inversion technique for a special class of distribution functions that only depend on energy and angular momentum through the combination

$Q \equiv \mathcal{E}-\frac{L^{2}}{2 r_{\mathrm{a}}^{2}}$,

with $r_{\mathrm{a}}$ the so-called anisotropy radius, with the additional condition that

$F(\mathcal{E}, L)=0 \quad$ for $Q<0$.

Such models correspond to an augmented density of the form

$\tilde{\rho}(\psi, r)=\left(1+\frac{r^{2}}{r_{\mathrm{a}}^{2}}\right)^{-1} f(\psi)$.

In this case, the fundamental integral Eq. (1) can be inverted in a similar way as the Eddington relation,

$$
F(\mathcal{E}, L)=\frac{1}{2 \sqrt{2} \pi^{2}} \frac{\mathrm{d}}{\mathrm{d} Q} \int_{0}^{Q} \frac{\mathrm{d} f}{\mathrm{~d} \psi} \frac{\mathrm{d} \psi}{\sqrt{Q-\psi}} .
$$

The anisotropy $\beta(r)$ for the Osipkov-Merritt models can be found by means of formula (11),

$\beta(r)=\frac{r^{2}}{r^{2}+r_{\mathrm{a}}^{2}}$

These models are hence isotropic in the center and completely radially anisotropic in the outer regions. The parameter $r_{\mathrm{a}}$ determines how soon the anisotropy turns from isotropic to radial. In particular, for $r_{\mathrm{a}} \rightarrow \infty, Q$ is nothing else than the binding energy, and the Osipkov-Merritt models reduce to the isotropic models.

The Osipkov-Merritt models were generalized by Cuddeford (1991), who considered models which correspond to an augmented density of the form

$\tilde{\rho}(\psi, r)=r^{-2 \beta_{0}}\left(1+\frac{r^{2}}{r_{\mathrm{a}}^{2}}\right)^{-1+\beta_{0}} f(\psi)$.

These models reduce to the Osipkov-Merritt models if we set $\beta_{0}=0$. Within this formalism, the distribution function can be calculated in a similar way as for the Osipkov-Merritt models. The distribution functions have the general form

$F(\mathcal{E}, L)=F_{0}(Q) L^{-2 \beta_{0}}$,

with the additional condition (43). The solution of the integral Eq. (1), valid for $\beta_{0}<1$, reads in this case

$$
\begin{aligned}
F(\mathcal{E}, L)= & \frac{2^{\beta_{0}}}{(2 \pi)^{3 / 2}} \frac{L^{-2 \beta_{0}}}{\Gamma\left(1-\beta_{0}\right) \Gamma(1-\theta)} \\
& \times \frac{\mathrm{d}}{\mathrm{d} Q} \int_{0}^{Q} \frac{\mathrm{d}^{m} f}{\mathrm{~d} \psi^{m}} \frac{\mathrm{d} \psi}{(Q-\psi)^{\theta}},
\end{aligned}
$$

where

$$
\begin{aligned}
m & =1+\operatorname{int}\left(\frac{1}{2}-\beta_{0}\right) \\
\theta & =\operatorname{frac}\left(\frac{1}{2}-\beta_{0}\right) .
\end{aligned}
$$

The most interesting cases are those where $\beta_{0}$ is either integer or half-integer. For integer values of $\beta_{0}$, the general formula $(49 \mathrm{abc})$ reduces to

$$
F(\mathcal{E}, L)=\frac{2^{\beta_{0}}}{2 \sqrt{2} \pi^{2}} \frac{L^{-2 \beta_{0}}}{\Gamma\left(1-\beta_{0}\right)} \frac{\mathrm{d}}{\mathrm{d} Q} \int_{0}^{Q} \frac{\mathrm{d}^{1-\beta_{0}} f}{\mathrm{~d} \psi^{1-\beta_{0}}} \frac{\mathrm{d} \psi}{\sqrt{Q-\psi}}
$$

For half-integer values of $\beta_{0}$, the integral Eq. (1) is a degenerate integral equation, which can be solved without a single integration (Dejonghe 1986; Cuddeford 1991) ${ }^{1}$,

$F(\mathcal{E}, L)=\frac{2^{\beta_{0}}}{(2 \pi)^{3 / 2}} \frac{L^{-2 \beta_{0}}}{\Gamma\left(1-\beta_{0}\right)}\left[\frac{\mathrm{d}^{\frac{3}{2}-\beta_{0}} f}{\mathrm{~d} \psi^{\frac{3}{2}-\beta_{0}}}\right]_{\psi=Q}$.

As for the Osipkov-Merritt models, the anisotropy of the Cuddeford models has a very simple functional form, which can be found through (11),

$\beta(r)=\frac{r^{2}+\beta_{0} r_{\mathrm{a}}^{2}}{r^{2}+r_{\mathrm{a}}^{2}}$.

1 Apparently, a factor $\Gamma\left(\frac{3}{2}-\beta_{0}\right)$ is missing in the denominator in formula (30) of Cuddeford (1991). 
They hence have an anisotropy $\beta_{0}$ in the center ${ }^{2}$, and become completely radially anisotropic in the outer regions. The anisotropy radius $r_{\mathrm{a}}$ is again a degree for how quick this transition takes place. In particular, for $r_{\mathrm{a}} \rightarrow \infty$, the Cuddeford models reduce to models with a constant anisotropy $\beta=$ $\beta_{0}$. Because the range of values for $\beta_{0}$ for which the inversion $(49 \mathrm{abc})$ is mathematically defined is only restricted by $\beta_{0}<1$, distribution functions can in principle be calculated with any degree of anisotropy in the center, ranging from very radial to extremely tangential. Whether these distribution functions correspond to physically acceptable solutions depends on the positivity, however.

\subsection{Hernquist models with increasing anisotropy}

\subsubsection{The distribution function}

For the Hernquist potential-density pair (2ab), the augmented density corresponding to the Cuddeford formalism is readily calculated. We obtain

$f(\psi)=\frac{1}{2 \pi}\left[1+\lambda\left(\frac{1-\psi}{\psi}\right)^{2}\right]^{1-\beta_{0}} \frac{\psi^{4-2 \beta_{0}}}{(1-\psi)^{1-2 \beta_{0}}}$,

where we have set $\lambda=1 / r_{\mathrm{a}}^{2}$. Combining this expression with the general Cuddeford solution (49abc) we can obtain distribution functions that self-consistently generate the Hernquist potential-density pair, and which have an arbitrary anisotropy in the center and a completely radial structure in the outer regions. In order to represent physically acceptable dynamical models, it is necessary that these distribution functions are positive over the entire phase space, i.e. $F(\mathcal{E}, L) \geqslant 0$ for $0 \leqslant Q \leqslant 1$. Before trying to actually calculate the distribution functions, it is useful to investigate which region in the $\left(\beta_{0}, \lambda\right)$ parameter space corresponds to physically acceptable distribution functions.

First of all, it is obvious that the models with $\beta_{0}>\frac{1}{2}$ will not correspond to non-negative distribution functions: the distribution function is already too radial for $\lambda=0$ (Sect. 4.2.1), and will become even more radial for larger $\lambda$. We can therefore limit the subsequent discussion to $\beta_{0} \leqslant \frac{1}{2}$. Now consider such a fixed value $\beta_{0}$, and consider all Cuddeford models corresponding to this central anisotropy. For $\lambda \rightarrow 0$, the Cuddeford model reduces to the model with constant anisotropy $\beta_{0}$, which is physically acceptable (Sect. 4.2.1). For $\lambda \rightarrow \infty$, the distribution function will only consist of radial orbits, for which the distribution function is not positive. It can therefore be expected that, for a given value of $\beta_{0} \leqslant \frac{1}{2}$, a range of $\lambda$ 's is allowed, starting from 0 up to a certain $\lambda_{\max }$.

Next, we have to investigate how $\lambda_{\max }$ varies with $\beta_{0}$, i.e. which anisotropy radii are allowed for a given central anisotropy? Distribution functions with a strong central tangential anisotropy and a small anisotropy radius are likely to be negative. Indeed, consider the orbital structure of such a galaxy. Because the outer regions of the galaxy $\left(r \gg r_{\mathrm{a}}\right)$ are strongly radially anisotropic, the vast majority of the stars there must be

\footnotetext{
2 This explains why we prefer the parameter $\beta_{0}$ above $\alpha=-\beta_{0}$ originally adopted by Cuddeford (1991).
}

Table 1. The range of anisotropy radii which give rise to a positive distribution function of the Cuddeford type, consistent with the Hernquist potential-density pair. For a given value of $\beta_{0}$, this range corresponds to $0 \leqslant \lambda \leqslant \lambda_{\max }$, or equivalently, to $r_{a, \min } \leqslant r_{\mathrm{a}} \leqslant \infty$.

\begin{tabular}{ccc}
\hline \hline$\beta_{0}$ & $\lambda_{\max }$ & $r_{\mathrm{a}, \min }$ \\
\hline$\leqslant-1.500$ & 0.000 & $\infty$ \\
-1.375 & 1.764 & 0.753 \\
-1.250 & 3.598 & 0.527 \\
-1.125 & 5.550 & 0.424 \\
-1.000 & 7.582 & 0.363 \\
-0.875 & 9.680 & 0.321 \\
-0.750 & 11.83 & 0.291 \\
-0.625 & 14.02 & 0.267 \\
-0.500 & 16.23 & 0.248 \\
-0.375 & 18.51 & 0.232 \\
-0.250 & 20.57 & 0.220 \\
-0.125 & 22.61 & 0.210 \\
0.000 & 24.42 & 0.202 \\
0.125 & 25.87 & 0.197 \\
0.250 & 26.70 & 0.194 \\
0.375 & 26.42 & 0.195 \\
0.500 & 24.00 & 0.204 \\
\hline
\end{tabular}

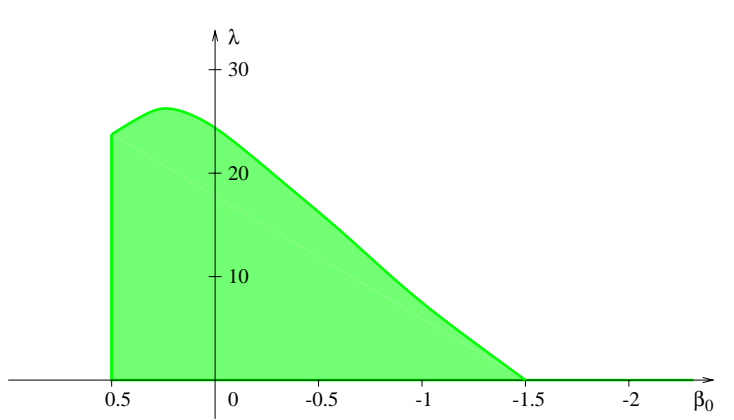

Fig. 3. The region in $\left(\beta_{0}, \lambda\right)$ space corresponding to a positive distribution function of the Cuddeford type, consistent with the Hernquist potential-density pair.

on nearly radial orbits. These stars also pass through the central regions, where they will contribute to the central density and radial velocity dispersion as well. The smaller the value of $r_{\mathrm{a}}$, i.e. the larger the value of $\lambda$, the stronger the contribution of stars on such nearly radial orbits. In order to create a core where the anisotropy is tangential, a large number of stars hence have to be added which move on tightly bound nearly circular orbits. But we are limited from keeping on adding such stars, because we cannot exceed the spatial density of the Hernquist profile, which has only a fairly weak $r^{-1}$ divergence. We therefore expect that no Cuddeford models will exist beyond a certain minimal $\beta_{0}$ (except for the degenerate case of the constant anisotropy models, which have no radial anisotropy at large radii). Moreover, it can be expected that for models with a tangential central anisotropy, the range of anisotropy radii is more restricted than for models with a radial or isotropic central anisotropy, i.e. that $\lambda_{\max }\left(\beta_{0}\right)$ is a increasing function of $\beta_{0}$.

By numerical evaluation of the integral in Eq. (49a), we calculated $\lambda_{\max }\left(\beta_{0}\right)$ for a number of values for $\beta_{0}$ (Table 1 ). The region in parameter space where the Cuddeford-Hernquist models are physical is shown in Fig. 3. Notice that all models 

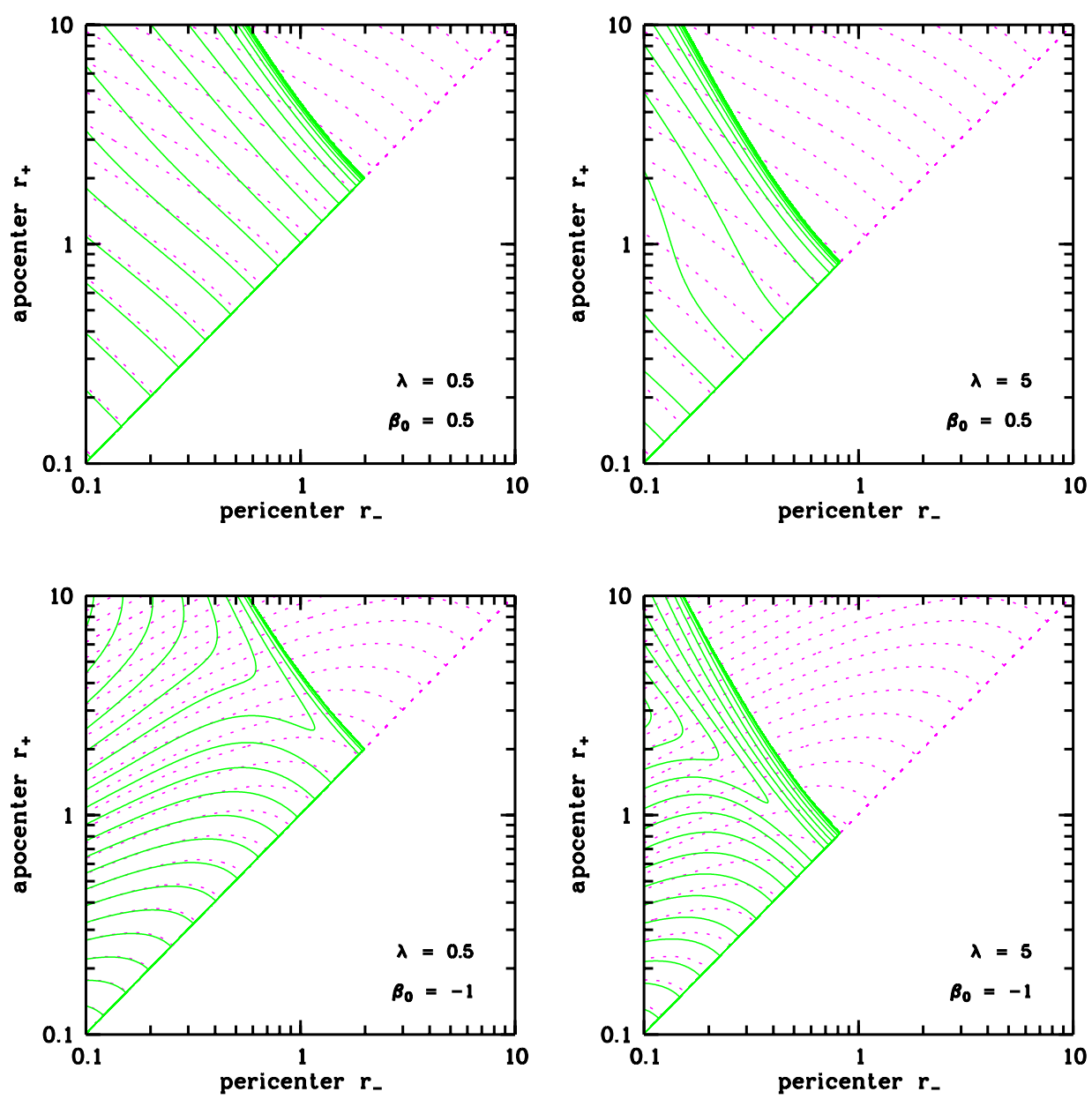

Fig. 4. Comparison of the distribution function corresponding to Hernquist models of the Cuddeford type and Hernquist models with a constant anisotropy. The distribution functions are represented as isoprobability contours in turning point space. The solid lines correspond to the Cuddeford distribution functions, with the parameters $\beta_{0}$ and $\lambda$ displayed in the down left corner of each diagram. The dotted lines represent the distribution functions of the corresponding Hernquist models with a constant anisotropy $\beta_{0}$.

with $\beta_{0} \leqslant-\frac{3}{2}$ and $\lambda>0$ are negative at some point in phase space and are thus unphysical: the Hernquist potential-density pair can support no (non-degenerate) distribution functions of the Cuddeford type with a central anisotropy $\beta_{0} \leqslant-\frac{3}{2}$.

We are primarily interested in those models where the distribution function can be expressed in terms of elementary functions. This is of course possible for all half-integer values of $\beta_{0}$, because the calculation of the distribution function involves no integrations. Also for the integer values of $\beta_{0}$, the distribution function can be calculated analytically, through the formula (50). Because of the limited region in $\left(\beta_{0}, \lambda\right)$ space where Cuddeford models are non-negative, this leaves us with four models with analytical distribution functions, corresponding to $\beta_{0}=\frac{1}{2}, 0,-\frac{1}{2}$ and -1 . The most simple of them is the case $\beta_{0}=\frac{1}{2}$, for which we obtain

$F(\mathcal{E}, L)=\frac{1}{4 \pi^{3}} \frac{1}{L} \frac{3 Q^{2}+\lambda\left(3 Q^{2}-5 Q+2\right)}{\sqrt{1+\lambda\left(\frac{1-Q}{Q}\right)^{2}}}$.
It is straightforward to check that this distribution function remains positive for $0 \leqslant \lambda \leqslant 24$, in agreement with the numerical result in Table 1 . For $\beta_{0}=0$, we recover the OsipkovMerritt model,

$$
\begin{aligned}
F(\mathcal{E}, L)= & \frac{1}{8 \sqrt{2} \pi^{3}}\left\{\frac{3 \arcsin \sqrt{Q}}{(1-Q)^{5 / 2}}\right. \\
& \left.+\sqrt{Q}(1-2 Q)\left[\frac{8 Q^{2}-8 Q-3}{(1-Q)^{2}}+8 \lambda\right]\right\},
\end{aligned}
$$

in agreement with Hernquist (1990). For the two other cases, $\beta_{0}=-\frac{1}{2}$ and $\beta_{0}=-1$, the distribution function can also be written in terms of elementary functions, but the expressions are somewhat more elaborate.

In Fig. 4 we show the distribution function of the Cuddeford type for four different models. The models on the top row have a radial central anisotropy, whereas those in the bottom panels have a tangential anisotropy in the center. The left and right column correspond to two different values of the anisotropy radius. The dotted distribution functions on 
the background are the distribution functions with a constant anisotropy $\beta_{0}$.

The character of the Cuddeford models can directly be interpreted from these figures. Compared to the constant anisotropy models, the Cuddeford models have a much larger fraction of stars on radial orbits, visible for both models with radial and tangential central anisotropy. The most conspicuous feature of each of the Cuddeford distribution functions is that the right part of the $\left(r_{-}, r_{+}\right)$diagram is completely empty, i.e. at large radii only the most radial orbits are populated, which is necessary to sustain the radial anisotropy. The boundary of the region in turning point space beyond which no orbits are populated can be calculated by translating the equation $Q=0$ in terms of the turning points $r_{-}$and $r_{+}$.

$\frac{r_{-}^{2} \psi\left(r_{-}\right)}{1+\lambda r_{-}^{2}}=\frac{r_{+}^{2} \psi\left(r_{+}\right)}{1+\lambda r_{+}^{2}}$.

When we substitute the Hernquist potential (2a), we can actually calculate the range of allowed orbits,

$$
\begin{aligned}
& 0 \leqslant r_{-} \leqslant r_{\mathrm{c}, \max } \\
& r_{-} \leqslant r_{+} \leqslant \frac{\left(1+r_{-}\right)+\sqrt{1+2 r_{-}+r_{-}^{2}+4 \lambda r_{-}^{3}}}{2 \lambda r_{-}^{2}},
\end{aligned}
$$

where $r_{\mathrm{c}, \max }$ represents the radius of the largest allowed circular orbit for a given $\lambda$,

$r_{\mathrm{c}, \max }=\frac{3^{1 / 3}+(9 \sqrt{\lambda}+\sqrt{81 \lambda-3})^{2 / 3}}{3^{2 / 3} \sqrt{\lambda}(9 \sqrt{\lambda}+\sqrt{81 \lambda-3})^{1 / 3}}$.

Obviously, the larger $\lambda$, the more restricted the range of allowed orbits, because the transition to radial anisotropy occurs at smaller radii for large values of $\lambda$. This can be seen when comparing the left and right panels of Fig. 4.

\subsubsection{The velocity dispersions}

In order to calculate the radial velocity dispersion associated with models of the Cuddeford type, we use the general formula (8a). After some manipulation, we obtain

$\sigma_{\mathrm{r}}^{2}(r)=\frac{r^{1-2 \beta_{0}}(1+r)^{3}}{\left(1+\lambda r^{2}\right)^{1-\beta_{0}}} \int_{r}^{\infty} \frac{\left(1+\lambda r^{\prime 2}\right)^{1-\beta_{0}} \mathrm{~d} r^{\prime}}{r^{\prime 1-2 \beta_{0}}\left(1+r^{\prime}\right)^{5}}$.

In general, this integral needs to be evaluated numerically, but for the four models with integer and half-integer values of $\beta_{0}$, it can be performed analytically. For example, for the OsipkovMerritt model $\beta_{0}=0$, we find

$$
\begin{aligned}
\sigma_{\mathrm{r}}^{2}(r)= & \frac{r(1+r)^{3}}{1+\lambda r^{2}} \ln \left(\frac{1+r}{r}\right) \\
& -\frac{r\left(25+52 r+42 r^{2}+12 r^{3}\right)-\lambda r(1+4 r)}{12(1+r)\left(1+\lambda r^{2}\right)},
\end{aligned}
$$

which reduces to the isotropic dispersion (19) for $\lambda=0$. For the other integer and half-integer values of $\beta_{0}$, the radial dispersion can also be expressed in terms of algebraic functions and logarithms, but the expressions are somewhat more elaborate.

In the top panels of Fig. 5 we plot the radial velocity dispersion profiles for Hernquist-Cuddeford models, for varying $\beta_{0}$ and varying $\lambda$ (left and right panels respectively). The behavior of $\sigma_{\mathrm{r}}$ as a function of $\beta_{0}$ is predictable. At small radii, the different models have a different behavior, with the largest dispersion for the most centrally radial models. At large radii they all have a similar, purely radial, orbital structure, and as a consequence their dispersion profiles all converge towards a single profile. This limiting profile is the radial velocity dispersion profile that corresponds to the (hypothetical) model with a completely radial orbital structure, which we can obtain by either setting $\beta=1$ in the expression (32), or setting $\beta_{0}=1$ in the expression (60),

$$
\sigma_{\mathrm{r}}^{2}(r)=\frac{1}{12} \frac{1+4 r}{r(1+r)}
$$

For a fixed central anisotropy, the behavior of the radial dispersion as a function of the anisotropy radius also follows a simple

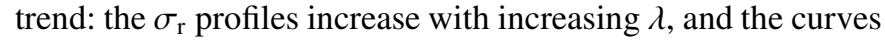
are all bounded by two limiting profiles: on the one hand the dispersion profile (32) of the constant anisotropy model (obtained by setting $\lambda=0$ ), and on the other hand the hypothetical dispersion profile (62) of the purely radial model (which corresponds to $\lambda \rightarrow \infty$ ). Dispersion profiles with large $\lambda$ will more quickly lean towards the purely radial profile than models with small $\lambda$, because the transition to a strongly radial anisotropy occurs at $r \sim r_{\mathrm{a}}=1 / \sqrt{\lambda}$.

The bottom panels of Fig. 5 show the line-of-sight velocity dispersion of the Hernquist-Cuddeford models. These profiles had to be calculated numerically. The dependence of the lineof-sight dispersion upon $\lambda$ and $\beta_{0}$ can be easily interpreted. In particular, the line-of-sight dispersion profiles of the Cuddeford models tend towards the line-of-sight dispersion profile of the hypothetical purely radial Hernquist model, which reads

$$
\begin{aligned}
& I(R) \sigma_{\mathrm{p}}^{2}(R)=\frac{R}{8}+\frac{1}{96 R}-\frac{1}{48 \pi\left(1-R^{2}\right)^{2}} \\
& \quad \times\left[R^{2}\left(20-29 R^{2}+12 R^{4}\right) X(R)+\left(2-7 R^{2}+4 R^{4}\right)\right]
\end{aligned}
$$

\section{Models with decreasing anisotropy}

\subsection{Background}

In order to construct dynamical models with a decreasing anisotropy, i.e. with a tangentially anisotropic halo, no special inversion techniques exist, such that we have to rely on the general formulae of Dejonghe (1986) to invert the fundamental integral Eq. (1). A disadvantage is that these formulae are numerically unstable. Their usefulness is therefore actually restricted to analytical models. But this is not straightforward: a direct application of the inversion formulae to an arbitrary analytical augmented density $\tilde{\rho}(\psi, r)$, even if its looks rather simple, can result in a cumbersome mathematical exercise, because the inversion formulae are quite elaborate.

A useful strategy to construct models with a tangential halo without the need to cope with the complicated general formulae, is to profit from the linearity of the integral Eq. (1). In particular, it is very interesting to generate augmented densities 

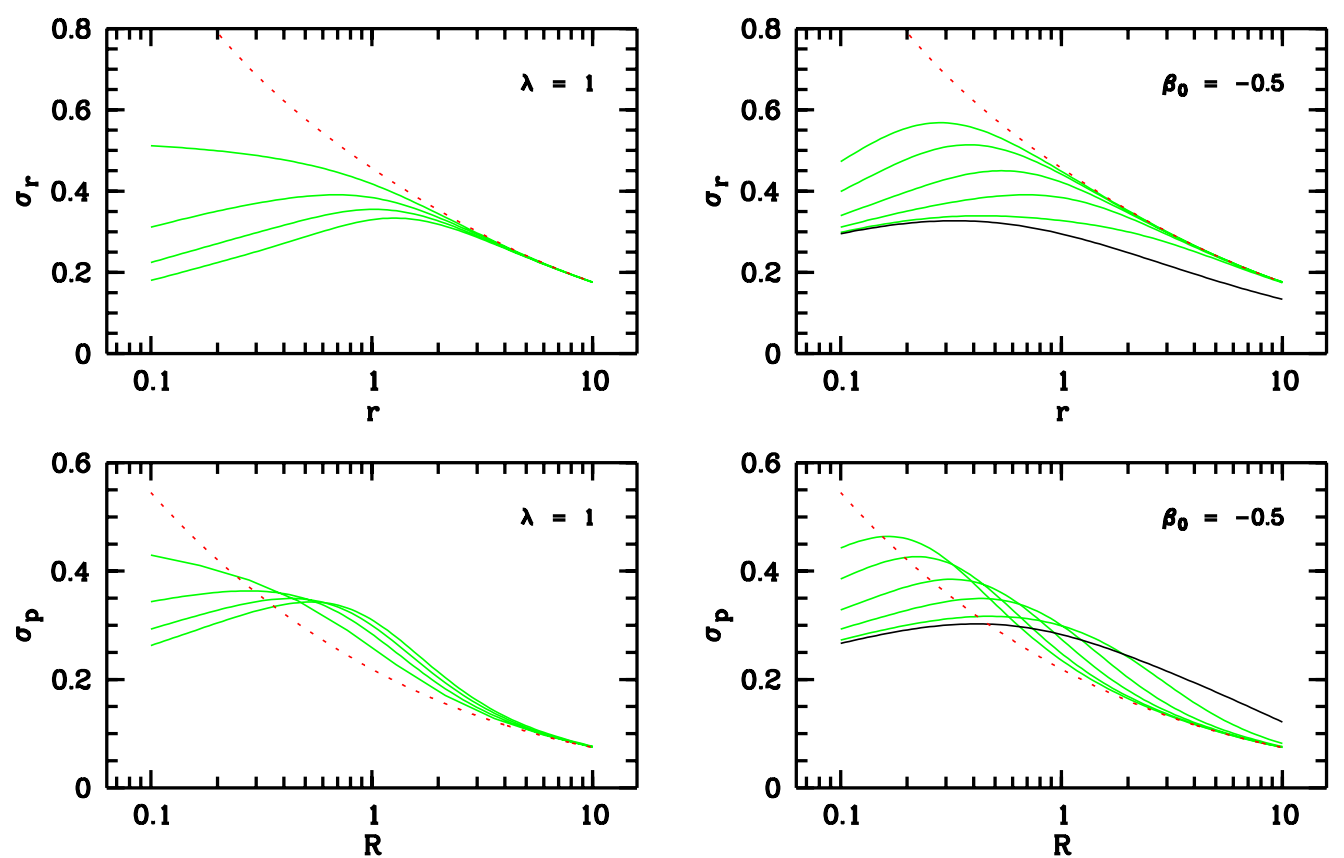

Fig. 5. The radial (upper panels) and line-of-sight (lower panels) velocity dispersion profiles of the Hernquist-Cuddeford models. The different curves in the two left panels correspond to models with the same anisotropy radius $\lambda=1$ (i.e. $r_{\mathrm{a}}=1$ ), but with a different central anisotropy parameter $\beta_{0}$ : plotted are $\beta=\frac{1}{2}, 0,-\frac{1}{2}$ and -1 . The dotted curves are the dispersion profiles of the (hypothetical) completely radial Hernquist model, which corresponds to $\beta_{0}=1$. The two panels on the right-hand side contain the dispersion profiles of models with the same central, slightly tangential, anisotropy parameter $\beta_{0}=-\frac{1}{2}$, but with a varying anisotropy radius. The various curves correspond to $\lambda=0$ (black solid line), $0.2,1,3,8$ and $\lambda_{\max } \equiv 16.23$. Again, the dotted curves are the dispersion profiles of the (hypothetical) completely radial Hernquist model, which corresponds to $\lambda \rightarrow \infty$.

$\tilde{\rho}(\psi, r)$, which can be expanded in a series of functions $\tilde{\rho}_{k}(\psi, r)$, which depend on $r$ only through a power law,

$\tilde{\rho}(\psi, r)=\sum_{k} \tilde{\rho}_{k}(\psi, r)=\sum_{k} f_{k}(\psi) r^{-2 \beta_{k}}$

Each of the augmented densities $\tilde{\rho}_{k}(\psi, r)$ corresponds to a dynamical model with a constant anisotropy $\beta_{k}$. Combining the linearity of the integral Eq. (1) with the results of Sect. 4.1, we find that the distribution function corresponding to the density (64) reads

$F(\mathcal{E}, L)=\sum_{k} F_{k}(\mathcal{E}, L)$

with

$$
\begin{aligned}
F_{k}(\mathcal{E}, L)= & \frac{2^{\beta_{k}}}{(2 \pi)^{3 / 2}} \frac{L^{-2 \beta_{k}}}{\Gamma\left(1-\beta_{k}\right) \Gamma\left(\frac{1}{2}+\beta_{k}\right)} \\
& \times \frac{\mathrm{d}}{\mathrm{d} \mathcal{E}} \int_{0}^{\mathcal{E}} \frac{\mathrm{d} f_{k}}{\mathrm{~d} \psi} \frac{\mathrm{d} \psi}{(\mathcal{E}-\psi)^{1 / 2-\beta_{k}}} .
\end{aligned}
$$

Equivalently, the moments of the distribution function can be derived from the series expansion.

\subsection{Hernquist models with decreasing anisotropy}

\subsubsection{Construction of the augmented density}

For every potential $\psi(r)$, we can create an infinite number of functions $Z(\psi, r)$ which satisfy the identity $Z(\psi(r), r) \equiv 1$.
For the Hernquist potential, we can easily create such a oneparameter family of functions $Z_{n}(\psi, r)$,

$Z_{n}(\psi, r)=[\psi(1+r)]^{n} \equiv 1$

with $n$ a natural number. If we multiply this family with the augmented density (27) of the constant anisotropy Hernquist models, we create a new two-parameter family of dynamical models, that will self-consistently generate the Hernquist potential-density pair,

$\tilde{\rho}(\psi, r)=\frac{1}{2 \pi} \frac{\psi^{4-2 \beta_{0}+n}}{(1-\psi)^{1-2 \beta_{0}}} \frac{(1+r)^{n}}{r^{2 \beta_{0}}}$.

Defining a new parameter $\beta_{\infty}=\beta_{0}-\frac{n}{2}$, we can write this augmented density also as

$\tilde{\rho}(\psi, r)=\frac{1}{2 \pi} \frac{\psi^{4-2 \beta_{\infty}}}{(1-\psi)^{1-2 \beta_{0}}} \frac{(1+r)^{2\left(\beta_{0}-\beta_{\infty}\right)}}{r^{2 \beta_{0}}}$.

Because we assumed that $n$ is a natural number, we can expand the binomial in the nominator of the density (67), and write it in the form (64), with

$$
\begin{aligned}
f_{k}(\psi) & =\frac{1}{2 \pi}\left(\begin{array}{l}
n \\
k
\end{array}\right) \frac{\psi^{4-2 \beta_{\infty}}}{(1-\psi)^{1-2 \beta_{0}}} \\
\beta_{k} & =\beta_{0}-\frac{k}{2},
\end{aligned}
$$

with $0 \leqslant k \leqslant n$. The reason why we chose $\beta_{0}$ and $\beta_{\infty}$ as parameters becomes clear when we look at the expression for the anisotropy corresponding to this family of density 


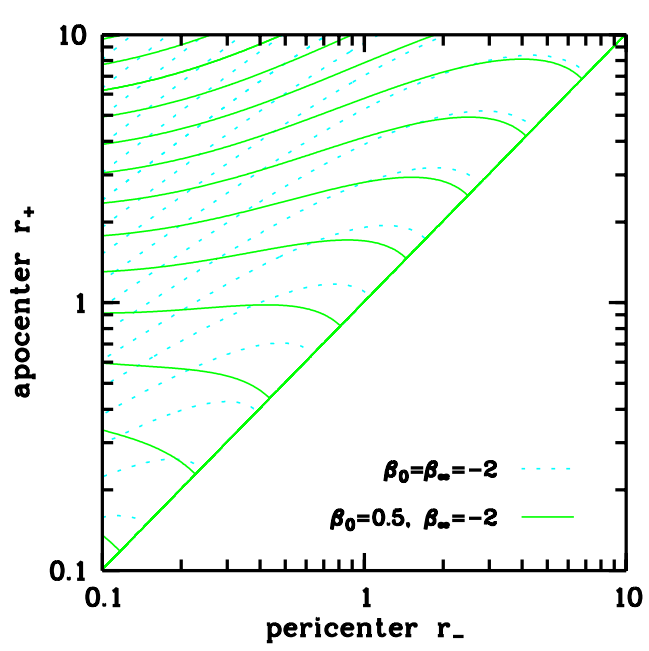

Fig. 6. Comparison of the distribution function corresponding to Hernquist model with increasing anisotropy with $\beta_{0}=\frac{1}{2}$ and $\beta_{\infty}=-2$, and the constant anisotropy model with $\beta=-2$. The distribution functions are represented as isoprobability contours in turning point space.

functions - for the moment being without bothering whether the density corresponds to a physically acceptable distribution function. By means of the formula (11), we obtain

$\beta(r)=\frac{\beta_{0}+\beta_{\infty} r}{1+r}$.

The anisotropy equals $\beta_{0}$ in the center and decreases to $\beta_{\infty}$ at large radii. Because $n$ can in principle assume any natural number, this family of augmented densities hence corresponds to dynamical models which can grow arbitrarily tangential in the outer regions. In particular, by setting $n=0$ we recover the models with constant anisotropy $\beta=\beta_{0}=\beta_{\infty}$ from Sect. 4.2.

\subsubsection{The distribution function}

We can calculate the distribution function of these models by applying the recipe (65b) to each of the components (69ab). We obtain after some algebra

$$
\begin{gathered}
F(\mathcal{E}, L)=\frac{2^{\beta_{0}}}{(2 \pi)^{5 / 2}} \Gamma\left(5-2 \beta_{\infty}\right) L^{-2 \beta_{0}} \mathcal{E}^{5 / 2-2 \beta_{\infty}+\beta_{0}} \\
\times \sum_{k=0}^{n}\left(\begin{array}{l}
n \\
k
\end{array}\right) \frac{1}{\Gamma\left(\frac{2+k}{2}-\beta_{0}\right) \Gamma\left(\frac{7}{2}-\frac{k}{2}-2 \beta_{\infty}+\beta_{0}\right)}\left(\frac{L}{\sqrt{2 \mathcal{E}}}\right)^{k} \\
\quad{ }_{2} F_{1}\left(5-2 \beta_{\infty}, 1-2 \beta_{0} ; \frac{7}{2}-\frac{k}{2}-2 \beta_{\infty}+\beta_{0} ; \mathcal{E}\right)
\end{gathered}
$$

This family of models is restricted by the condition $\beta_{0} \leqslant \frac{1}{2}$, because for higher values of $\beta_{0}$ the distribution function becomes negative. For all half-integer and integer values of $\beta_{0}$ (and therefore also of $\beta_{\infty}$ ), this expression can be written in terms of elementary functions, very analogous with the distribution functions of the constant anisotropy models: the expression contains integer and half-integer powers of $\mathcal{E}$ and $1-\mathcal{E}$ and a factor $\arcsin \sqrt{\mathcal{E}}$. The models characterized by $\beta_{0}=\frac{1}{2}$ are of a particular kind. The hypergeometric functions in Eq. (71) disappears for $\beta_{0}=\frac{1}{2}$, such that the distribution function can be written as a finite power series of $\sqrt{\mathcal{E}}$ and $L$.
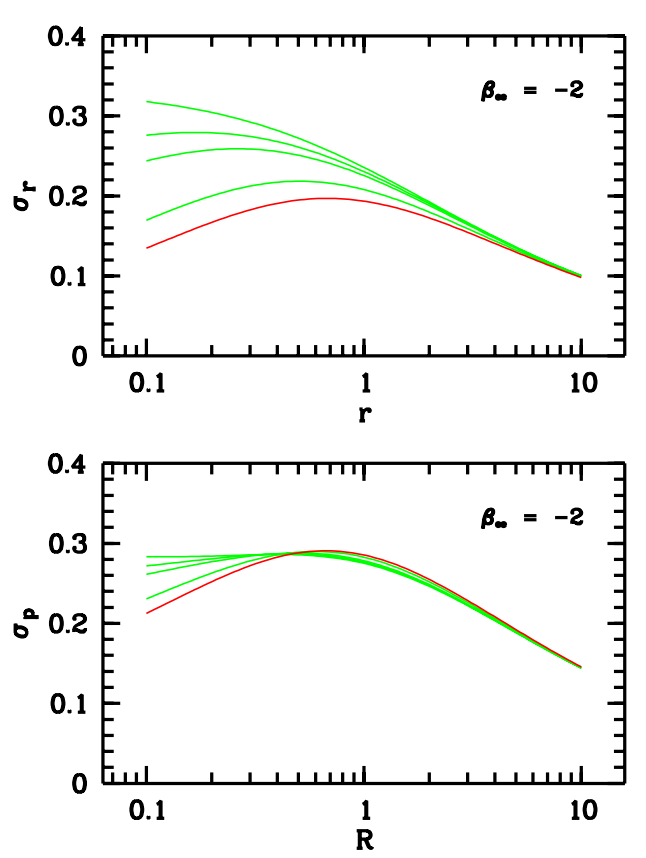

Fig. 7. The radial (upper panel) and line-of-sight (lower panel) velocity dispersion profiles of Hernquist models with a decreasing anisotropy. All models have the same tangential outer anisotropy $\beta_{\infty}=-2$, but they have a different central anisotropy parameter $\beta_{0}$ : plotted are $\beta_{0}=\frac{1}{2}, 0,-\frac{1}{2},-1$ and -2 (black line).

An interesting characteristic of these models is revealed when we look at the asymptotic behavior of the distribution function at large radii, i.e. for $\mathcal{E} \rightarrow 0$. The term corresponding to $k=n$ will contribute the dominant term in the sum (71), such that we obtain

$$
\begin{aligned}
F(\mathcal{E}, L) \approx \frac{2^{\beta_{\infty}}}{(2 \pi)^{5 / 2}} & \frac{\Gamma\left(5-2 \beta_{\infty}\right)}{\Gamma\left(1-\beta_{\infty}\right) \Gamma\left(\frac{7}{2}-\beta_{\infty}\right)} \\
& \times L^{-2 \beta_{\infty}} \mathcal{E}^{5 / 2-\beta_{\infty}}+\cdots
\end{aligned}
$$

This expansion is at first order independent of $\beta_{0}$, such that all models with the same $\beta_{\infty}$ will have a similar behavior at large radii. In particular, all distribution functions corresponding to a particular $\beta_{\infty}$ will at large radii behave as the Hernquist model with constant anisotropy $\beta=\beta_{\infty}$. This is illustrated in Fig. 6, where we compare the distribution function of a model with a radial core and a tangential halo with the constant anisotropy model that has the corresponding tangential anisotropy. At small radii, the difference between both distribution functions is obvious: the former one has more stars on radial orbits, whereas the latter prefers to populate circular-like orbits. At large radii, however, the isoprobability contours of both models agree very well.

Finally, notice that there is no analogue for this behavior at small radii: not all models with a fixed $\beta_{0}$ will have a similar behavior for $\mathcal{E} \rightarrow 1$, i.e. at small radii.

\subsubsection{The velocity dispersions}

In order to calculate the velocity dispersion profiles of the models of this type, we have various possibilities. We can either 
calculate the dispersion for each of the $n$ terms (69a) through formula (26) and sum the results, or directly apply the general recipe (8ab) on the expression (68). In either case, we obtain an expression very akin to the expression (32) of the models with constant anisotropy,

$\sigma_{\mathrm{r}}^{2}(r)=r^{1-2 \beta_{0}}(1+r)^{3+2 \beta_{\infty}-2 \beta_{0}} \mathrm{~B}_{\frac{1}{1+r}}\left(5-2 \beta_{\infty}, 2 \beta_{0}\right)$.

This expression can be written in terms of elementary functions for all $\beta_{0}$ with $4 \beta_{0}$ an integer (and hence also $4 \beta_{\infty}$ an integer).

Not as a surprise, the asymptotic expressions for $\sigma_{\mathrm{r}}^{2}(r)$ for $r \gg 1 \mathrm{read}$

$\sigma_{\mathrm{r}}^{2}(r) \approx \frac{1}{5-2 \beta_{\infty}} \frac{1}{r}+\cdots$

i.e. they are similar to the corresponding expansions of the constant anisotropy models with $\beta=\beta_{\infty}$. This behavior is illustrated in the upper panel of Fig. 7 , where we plot the radial velocity dispersion profile for a set of models with varying $\beta_{0}$ and a fixed $\beta_{\infty}$. At small radii, the models have different profiles (those with the most radial anisotropy have the largest values of $\sigma_{\mathrm{r}}$ ), but at large radii, they all converge towards a common asymptotic expansion.

The calculation of the line-of-sight velocity dispersion is also similar to the case of constant anisotropy. It is found that $\sigma_{\mathrm{p}}(R)$ can be written in terms of elementary functions for all integer and half-integer values of $\beta_{0}$, and that the asymptotic behavior for $R \gg 1$ reads

$\sigma_{\mathrm{p}}^{2}(R) \approx \frac{8}{15 \pi}\left(\frac{5-4 \beta_{\infty}}{5-2 \beta_{\infty}}\right) \frac{1}{R}+\cdots$,

which is at first order independent of $\beta_{0}$. An illustration is shown in the bottom panel of Fig. 7.

\section{Conclusions}

Three new families of anisotropic dynamical models have been presented that self-consistently generate the Hernquist potential-density pair. For all models, in particular for the Cuddeford models of Sect. 5, we checked the conditions on the adopted parameters such that the distribution is positive, and hence physically acceptable, in phase space.

They host a wide variety of orbital structures: in general, the models presented can have an arbitrary central anisotropy, and a outer halo with the same anisotropy, a purely radial orbital structure, or an arbitrary, but more tangential, anisotropy. In order to produce models that have an arbitrary anisotropy in the central regions, and a more radial, but not purely radial, anisotropy at large radii, the most cost-effective way seems to construct a linear combination of a number of 'component' dynamical models, such as the ones presented here. This technique has been adopted for several years in the QP formalism (Dejonghe 1989, for an overview see Dejonghe et al. 2001), where most of the components in the program libraries have an intrinsically tangential orbital structure.

For all of the presented models, we have analytical expressions for the distribution function and the velocity dispersions in terms of elementary functions. They are hence ideal tools for a wide range of applications, for example to generate the initial conditions for $N$-body or Monte Carlo simulations. At this point, a number of remarks are appropriate.

First, very few elliptical galaxies are perfectly spherical; actually, various observational and theoretical evidence suggests that many elliptical galaxies are at least moderately triaxial (Dubinski \& Carlberg 1991; Hernquist 1993; Tremblay \& Merritt 1995; Bak \& Statler 2000). Unfortunately, an extension of the presented techniques to construct analytical axisymmetric or triaxial systems is not obvious, because the internal dynamics of such stellar systems is much more complicated than in the spherical case. Nevertheless, our models can be used as an onset to construct numerical axisymmetric of triaxial distribution functions with different internal dynamical structures, for example by the adiabatic squeezing technique presented by Holley-Bockelmann et al. (2001).

Second, the models presented here are self-consistent models, whereas it is nowadays believed that most elliptical galaxies contain dark matter, either in the form of a central black hole (Merritt \& Ferrarese 2001 and references therein) and/or a dark halo (Kronawitter et al. 2000; Magorrian \& Ballantyne 2001). When constructing dynamical models with dark matter, an extra component must be added to the gravitational potential. For example, Ciotti (1996) constructed analytical two-component models in which both the stellar and dark matter components have a Hernquist density profile and an Osipkov-Merritt type distribution function. The models presented in this paper can also be extended to contain a dark halo or a central black hole. Indeed, the adopted inversion techniques are perfectly suitable for this, because the augmented density functions $\tilde{\rho}(\psi, r)$ do not necessarily need to satisfy the self-consistency condition (5). Adding an extra term to the potential does not conceptually change the character of the inversion, but it might complicate the mathematical exercise.

Third, we have not discussed stability issues for the presented models. The study of the stability of anisotropic stellar systems is difficult, and a satisfactory criterion can not easily be given. For stability against radial perturbations, we can apply the sufficient criterions of Antonov (1962) or Dorémus \& Feix (1973), but numerical simulations have shown that these criteria are rather crude (Dejonghe \& Merritt 1988; Meza \& Zamorano 1997). Moreover, the only instability that is thought to be effective in realistic galaxies is the so-called radial orbit instability, an instability that drives galaxies with a large number of radial orbits to forming a bar (Hénon 1973; Palmer \& Papaloizou 1987; Cincotta et al. 1996). The behavior of galaxy models against perturbations of this kind can only be tested with detailed $N$-body simulations or numerical linear stability analysis. Meza \& Zamorano (1997) used $N$-body simulations to investigate the radial orbit instability for a number of spherical models of the Osipkov-Merritt type, including the Hernquist model. They found that the models are unstable for $r_{\mathrm{a}} \lesssim 1$, which significantly restricts the set of models that correspond to positive distribution functions (see Table 1). It would be interesting to extend this investigation to the three families of Hernquist models presented in this paper, but this falls beyond the scope of this paper. 
Acknowledgements. The authors are grateful to Andrés Meza for a careful check on the formulae derived in this paper. Fortran codes to evaluate the internal and projected dynamics of the presented models are available from the authors.

\section{References}

Abramowitz, M., \& Stegun, I. A. 1972, Handbook of Mathematical Functions (Dover Publication Inc., New York)

Antonov, V. A. 1962, Vestnik Leningrad University, 19, 96 (Trans. in Princeton Plasma Physics Lab. Rept. PPI-Trans-1)

Bak, J., \& Statler, T. 2000, AJ, 120, 110

Binney, J., \& Tremaine, S. 1987, Galaxy Dynamics (Princeton University Press, Princeton)

Carollo, C. M., de Zeeuw, P. T., \& van der Marel, R. P. 1995, MNRAS, 276,1131

Ciotti, L. 1991, A\&A, 249, 99

Ciotti, L. 1996, ApJ, 471, 68

Ciotti, L., \& Lanzoni, B. 1997, A\&A, 321, 724

Cincotta, P. M., Nunez, J. A., \& Muzzio, J. C. 1996, ApJ, 456, 274

Cuddeford, P. 1991, MNRAS, 253, 414

Dejonghe, H. 1986, Phys. Rep., 133, 217

Dejonghe, H. 1987, MNRAS, 224, 13

Dejonghe, H. 1989, ApJ, 343, 113

Dejonghe, H., \& Merritt, D. 1988, ApJ, 328, 93

Dejonghe, H., Baes, M., De Bruyne, V., et al. 2001, in Stellar

Dynamics: From Classic to Modern, ed. L. P. Osipkov, \& I. I. Nikiforov (Saint-Petersburg State University), p. 292

Dehnen, W. 1993, MNRAS, 265, 250

de Vaucouleurs, G. 1948, Ann. Astrophys., 11, 247
Dorémus, J. P., \& Feix, M. R. 1973, A\&A, 29, 401

Dubinski, J., \& Carlberg, R. 1991, ApJ, 378, 496

Eddington, A. S. 1916, MNRAS, 76, 572

Fricke, W. 1951, Astron. Nachr., 280, 193

Gebhardt, K., Richstone, D., Ajhar E. A., et al. 1996, AJ, 112, 105

Hénon, M. 1959, Ann. Astrophys., 22, 126

Hénon, M. 1960, Ann. Astrophys., 23, 474

Hénon, M. 1973, A\&A, 24, 229

Hernquist, L. 1990, ApJ, 356, 359

Hernquist, L. 1993, ApJ, 409, 548

Holley-Bockelmann, K., Mihos, J. C., Sigurdsson, S., \& Hernquist, L. 2001, ApJ, 549, 862

Jaffe, W. 1983, MNRAS, 202, 995

Kronawitter, A., Saglia, R. P., Gerhard, O., \& Bender R. 2000, A\&AS, 144,53

Lauer, T. R., Ajhar, E. A., Byun, Y., Dressler, A., \& Faber, S. M. 1995, AJ, 110, 2622

Magorrian, J., \& Ballantyne, D. 2001, MNRAS, 322, 702

Mazure, A., \& Capelato, H. V. 2002, A\&A, 383, 384

Merritt, D. 1985, AJ, 90, 1027

Merritt, D., \& Ferrarese, L. 2001, MNRAS, 320, L30

Meza, A., \& Zamorano, N. 1997, ApJ, 490, 136

Osipkov, L. P. 1979, Sov. Astron. Lett., 5, 42

Palmer, P. L., \& Papaloizou, J. 1987, MNRAS, 224, 1043

Plummer, H. C. 1911, MNRAS, 71, 460

Richstone, D. O., \& Tremaine, S. 1984, ApJ, 286, 27

Sérsic, J. L. 1968, Atlas de Galaxias Australes, Cordoba: Observatorio Astronómico

Tremaine, S., Richstone, D. O., Byun, Y.-I., et al. 1994, AJ, 107, 634

Tremblay, B., \& Merritt, D. 1995, AJ, 110, 1039 